\title{
Near-Threshold Cross Sections for Electron and Positron Impact Ionization of Atomic Hydrogen
}

\author{
I. Bray, ${ }^{1}$ A. W. Bray, ${ }^{2}$ D. V. Fursa, ${ }^{1}$ and A. S. Kadyrov ${ }^{1}$ \\ ${ }^{1}$ Curtin Institute for Computation and Department of Physics and Astronomy, Curtin University, \\ GPO Box U1987, Perth, Western Australia 6845, Australia \\ ${ }^{2}$ Research School of Physics and Engineering, The Australian National University, \\ Canberra, Australian Capital Territory 0200, Australia
}

(Received 11 September 2018; published 16 November 2018)

\begin{abstract}
Using classical arguments Wannier [Phys. Rev. 90, 817 (1953)] proposed an electron-impact ionization cross section for neutral atoms to behave as $E^{1.127}$, where $E$ is the excess energy above threshold. Using similar arguments Klar [J. Phys. B 14, 4165 (1981)] obtained $E^{2.65}$ to be the corresponding threshold law for positron impact. Recently, Babij et al. [Phys. Rev. Lett. 120, 113401 (2018)] measured near-threshold positron-impact breakup behavior to be similar to that expected for electrons. Using the convergent closecoupling method for the atomic hydrogen target, we examine cross sections at near-threshold energies for electron and positron impact. Contrary to the experiment, the calculated cross sections are found to behave differently for the two projectiles and consistently with the aforementioned threshold laws, despite the entirely quantum nature of these problems. For electron impact, the threshold behavior holds while the total electron spin asymmetry remains constant, whereas for positron scattering the threshold law holds for breakup while the positronium-formation component of the ionization cross section remains constant.
\end{abstract}

DOI: 10.1103/PhysRevLett.121.203401

The threshold behavior of cross sections $\sigma_{I}$ for electronimpact ionization of atoms has been of extraordinary interest following the classical approach to the problem proposed by Wannier [1]. He treated the electrons as classical particles moving in opposite directions with equal speeds on either side of the residual ion of charge $Z$. The arguments are generally target independent, with the derived threshold law being

$$
\sigma_{I} \propto E^{\mu / 2-\frac{1}{4}}, \quad \text { with } \quad \mu=\frac{1}{2} \sqrt{\frac{100 Z-9}{4 Z-1}}
$$

where $E$ is the excess energy above threshold (total energy). For electron-impact ionization of neutral atoms $\sigma_{I} \propto E^{1.127}$.

In the case of positron-impact ionization, using similar arguments to Wannier, the ionization (excluding positronium formation) cross section threshold behavior was predicted to be $E^{2.65}$ by Klar [2], and was further supported and expanded upon by Ihra et al. [3]. Thus, the cross section behavior is predicted to be much more suppressed near threshold in comparison to the electron case. Yet recently Babij et al. [4] performed near-threshold measurements and suggested that the positron-impact behavior is much the same as for electron impact. It is particularly challenging extracting threshold behavior from experiments, as also discussed by Ashley et al. [5], but the rather large variation between the measured and the predicted power law is what motivates the present study.
There have been many studies of threshold laws, see major reviews of Rau [6] and Rost [7], for example. As far as we are aware, the general consensus is that threshold laws derived from Wannier arguments are valid, with some notable exceptions $[8,9]$. The latter predict the same behavior for both electron and positron impact [9]. From our perspective the interest in threshold laws is more of a practical nature. It is very helpful to have a way to extrapolate to zero from $a b$ initio calculations at some small $E$, particularly when Eq. (1) is so generally applicable. Yet, we cannot help but wonder why any classical argument for Coulomb three-body problems may be valid at any low energy, or what the range of validity might be for practical purposes.

The threshold laws are target independent since the Coulomb interaction of the three charged particles is assumed to be the most dominant at low energies. We will use the atomic hydrogen target to study this problem. The electron-impact problem is fully quantum mechanical due to the importance of the total electron spin $S$ with spin-dependent cross sections $\sigma_{I}^{(S)}$. For positron impact, a unitary treatment is required due to the very large competing ionization channel of positronium (Ps) formation in any of countably infinite bound states. A fully $a b$ initio computational approach is required to determine which threshold behavior is correct, and its range of validity.

In a nonrelativistic quantum mechanical treatment, electron-impact ionization of hydrogen breaks up into 
two independent total electron spins $S=0,1$ with the cross section and spin asymmetry defined by

$\sigma_{I}=\frac{\sigma_{I}^{(S=0)}+3 \sigma_{I}^{(S=1)}}{4}, \quad A_{I}=\frac{\sigma_{I}^{(S=0)}-\sigma_{I}^{(S=1)}}{\sigma_{I}^{(S=0)}+3 \sigma_{I}^{(S=1)}}$.

For the $\mathcal{L}=0$ partial wave of the total orbital angular momentum, Peterkop [10] showed that $\sigma_{I}^{(S=0)}$ followed Eq. (1), but that $\sigma_{I}^{(S=1)}$ was much more suppressed by a factor of three in the power. This was confirmed by Bartlett et al. [11] using $a b$ initio calculations. Hence, for $\mathcal{L}=0$ the spin-weighted cross section $\sigma_{I}$ satisfies Eq. (1) only due to the overwhelming dominance of $\sigma_{I}^{(S=0)}$, with the corresponding spin asymmetry $A_{I} \approx 1$ near threshold. However, for the full problem the experimental data [12,13] suggest that near threshold $A_{I} \approx 0.5$ and so $\sigma_{I}^{(S=0)} \approx 5 \sigma_{I}^{(S=1)}$. Clearly, the relationship between the validity of Eq. (1) and the spin asymmetry deserves attention.

To study near threshold ionization we use the convergent close-coupling (CCC) method for electron scattering [14] and its two-center variant for positron scattering [15]. The most recent review of CCC application to collision with atoms and molecules may be found in Ref. [16]. The CCC approach relies on the complete Laguerre basis $N_{l}$ with exponential fall-off parameter $\lambda_{l}$ to diagonalize the target ( $T=\mathrm{H}$ or Ps) Hamiltonian

$$
\left\langle\phi_{f}\left|H_{T}\right| \phi_{i}\right\rangle=\epsilon_{f} \delta_{f i},
$$

to obtain a set of pseudostates $\phi_{n}, n=1, \ldots, N_{l}$, for each target orbital angular momentum $l \leq l_{\max }$. With increasing $N_{l}$ the negative-energy states converge to the discrete eigenstates of the target, while the positive-energy states provide an increasingly dense discretization of the target continuum. Ionization processes are identified with excitation of the positive-energy pseudostates, which yield accurate fully differential cross sections [17]. In the case of positron scattering, Eq. (3) is used separately for the atomic and the Ps states.

Having obtained the Laguerre-based states the resulting close-coupling equations are formed in momentum space for the transition amplitudes as

$$
\begin{aligned}
\left\langle\boldsymbol{k}_{f} \boldsymbol{\phi}_{f}\left|T_{S}\right| \phi_{i} \boldsymbol{k}_{i}\right\rangle & =\left\langle\boldsymbol{k}_{f} \boldsymbol{\phi}_{f}\left|V_{S}\right| \phi_{i} \boldsymbol{k}_{i}\right\rangle \\
& +\sum_{n=1}^{N_{\mathrm{H}}+N_{\mathrm{Ps}}} \int d^{3} k \frac{\left\langle\boldsymbol{k}_{f} \boldsymbol{\phi}_{f}\left|V_{S}\right| \phi_{n} \boldsymbol{k}\right\rangle\left\langle\boldsymbol{k} \boldsymbol{\phi}_{n}\left|T_{S}\right| \phi_{i} \boldsymbol{k}_{i}\right\rangle}{E+i 0-\epsilon_{n}-\varepsilon_{k}} .
\end{aligned}
$$

Here $E$ is the total energy, $N_{\mathrm{H}}$ is the total number of $\mathrm{H}$ states and $N_{\mathrm{Ps}}$ is the total number of Ps states (positron scattering only).
For electron scattering, $S=0,1$ is the total electron spin with the direct $(D)$ and exchange $(E)$ potentials yielding $V_{S}=V_{D}+(-1)^{S} V_{E}$. Solution of Eq. (4) is performed following partial wave expansion in total orbital angular momentum $\mathcal{L}$ utilizing numerical quadrature to manage the singularity in the denominator. Cross sections are obtained from

$$
\sigma_{f i}^{(S)} \equiv \frac{k_{f}}{k_{i}}\left|\left\langle\boldsymbol{k}_{f} \boldsymbol{\phi}_{f}\left|T_{S}\right| \phi_{i} \boldsymbol{k}_{i}\right\rangle\right|^{2} .
$$

Our interest is in

$$
\sigma_{I}^{(S)}=\sum_{f: 0<\epsilon_{f}<E} \sigma_{f i}^{(S)}
$$

for initial state $i$ being the ground state of atomic hydrogen. The $\sigma_{I}^{(S)}$ need to be convergent with increasing bases sizes $N_{l}$ and $l_{\max }$. For ease of convergence studies we set $\lambda_{l}^{\mathrm{H}}=1$, $\lambda_{l}^{\text {Ps }}=0.5, N_{l}=N_{0}-l$, and label the calculations by $\operatorname{CCC}\left(N_{0 l_{\max }}\right)$.

One of the first major successes of the CCC method was the reproduction of the $e^{-}-\mathrm{H}$ total ionization cross sections and spin asymmetries on a broad energy range [18]. Since that time we have addressed the formal scattering theory with long ranged potentials [19] and explained why CCC and other computationally intensive methods are able to obtain fully differential ionization cross sections [20]. The original calculations [18] could be labeled as $\operatorname{CCC}\left(10_{3}\right)$ and $\operatorname{CCC}\left(13_{2}\right)$, but were too small to be accurate at energies $0<E<5 \mathrm{eV}$. Now we have the computational resources to extend such calculations to very small $E$.

In Fig. 1 we give the results of $\mathrm{CCC}\left(30_{3}\right)$ calculations at energies from 0.1 to $1000 \mathrm{eV}$ and compare with experiment and the Wannier threshold law Eq. (1). The cross section presentation is on a log-log scale in order to emphasise the lowest energies. In addition to the $\operatorname{CCC}\left(30_{3}\right)$ calculation performed over the entire energy range, also presented are $\operatorname{CCC}\left(80_{3}\right)$ calculations up to $2 \mathrm{eV}$. We see outstanding agreement between the CCC calculations and the cross section and spin asymmetry measurements. It appears that the spin asymmetry parameter remains approximately constant at 0.5 up to around $2 \mathrm{eV}$ above threshold, and then begins to diminish slowly. This means that $\sigma_{I}^{(S=0)} \approx$ $5 \sigma_{I}^{(S=1)}$ below $2 \mathrm{eV}$, allowing for a spin-independent threshold law to be potentially valid. The cross section figure shows that the Wannier threshold law, when normalized at $1 \mathrm{eV}$, remains in good agreement with experiment and the CCC calculations up to about $2 \mathrm{eV}$. Furthermore, as we increase $N_{0}$ to 80 the CCC calculations yield improved agreement with the threshold law to lower energies.

It is also interesting to ask why $\sigma_{I}^{(S=0)} \approx 5 \sigma_{I}^{(S=1)}$ near threshold. We are only able to explain why $\sigma_{I}^{(S=1)} \ll \sigma_{I}^{(S=0)}$. 

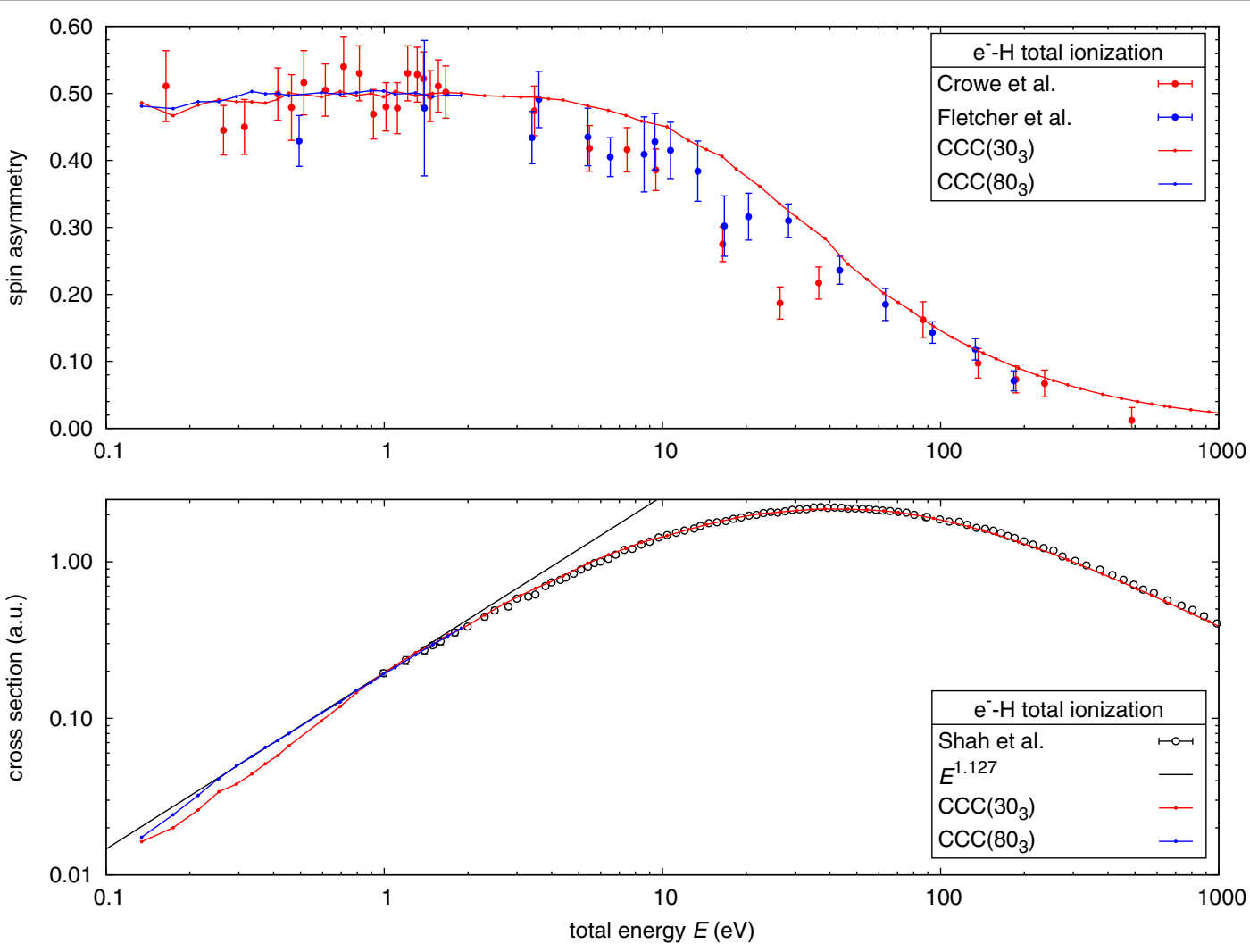

FIG. 1. Total ionization cross section (bottom) and spin asymmetry (top) of electron scattering on atomic hydrogen. The present CCC calculations, performed at specified points connected with straight lines to guide the eye, are labeled by $\mathrm{CCC}\left(N_{0 l_{\max }}^{\mathrm{H}}\right)$ with $\mathrm{Laguerre}$ basis size $N_{l}=N_{0}-l$ for orbital angular momentum $l \leq l_{\max }=3$. The calculations with $N_{0}=30$ extend over the full energy range. Those with $N_{0}=80$ extend to $2 \mathrm{eV}$ above threshold. The Wannier [1] threshold law has been normalized at $1 \mathrm{eV}$ for best visual fit. The experimental data are due to Shah et al. [21], Fletcher et al. [12], and Crowe et al. [13].

This is due to the fact that the $S=1$ potential matrix elements are a difference of the direct and exchange terms. At small $E$ the two outgoing electrons have similar small energies and so the matrix elements are of commensurate magnitude. This makes $V_{S=1} \ll V_{S=0}$ when solving Eq. (4).

The near threshold spin asymmetry behavior observed here for atomic hydrogen is similar to that for the quasione-electron atoms or ions of the Li-like sequence [22], sodium and potassium [23]. For such targets the spin asymmetry tends to a nonzero constant at threshold, which varies with the target. In all cases the triplet component is smaller than the singlet, but not negligible.

Having demonstrated that the CCC method is suitable for studying threshold laws and able to provide convergent results down to very low energies for electron impact, we now consider the positron-impact ionization problem. For positrons the ionization process has two components, Ps formation and breakup (three free particles in the final state). The threshold law of Klar [2] applies only to the breakup component. Note that the Ps-formation threshold is $6.8 \mathrm{eV}$ lower than the breakup threshold.

Convergence studies in two-center problems have to be done for both centers, presently for both $\mathrm{H}$ and
Ps. Such problems are inherently ill conditioned due to the usage of two (truncated) complete bases. However, due to unitarity of the close-coupling formalism there is no double counting. These issues have been discussed at length with two-center and one-center approaches to the problem being important internal consistency checks [24]. This ill-conditioning is such that when particularly small results are required an analytic approach to the treatment of the Green's function singularity in Eq. (4) has greatest accuracy [25], and is used here at the lower energies.

There are several ways that we can demonstrate convergence. There is a symmetric approach where $N_{0}^{\mathrm{H}}=N_{0}^{\mathrm{Ps}}$ and $l_{\max }^{\mathrm{H}}=l_{\max }^{\mathrm{Ps}}$. This has already been shown to work well for the corresponding simpler $\mathcal{L}=0$ problem [26]. It was found that the model problem satisfied the threshold law of Klar [2] and its modified form derived by Ihra et al. [3]. In symmetric approaches there are contributions to the breakup cross section that come from the positive-energy atomic and Ps states. Unfortunately, for full calculations such an approach has proved to be too ill conditioned to yield reliable results for $E<1 \mathrm{eV}$. Instead, we limit the Ps states to just those with negative energies, with the 


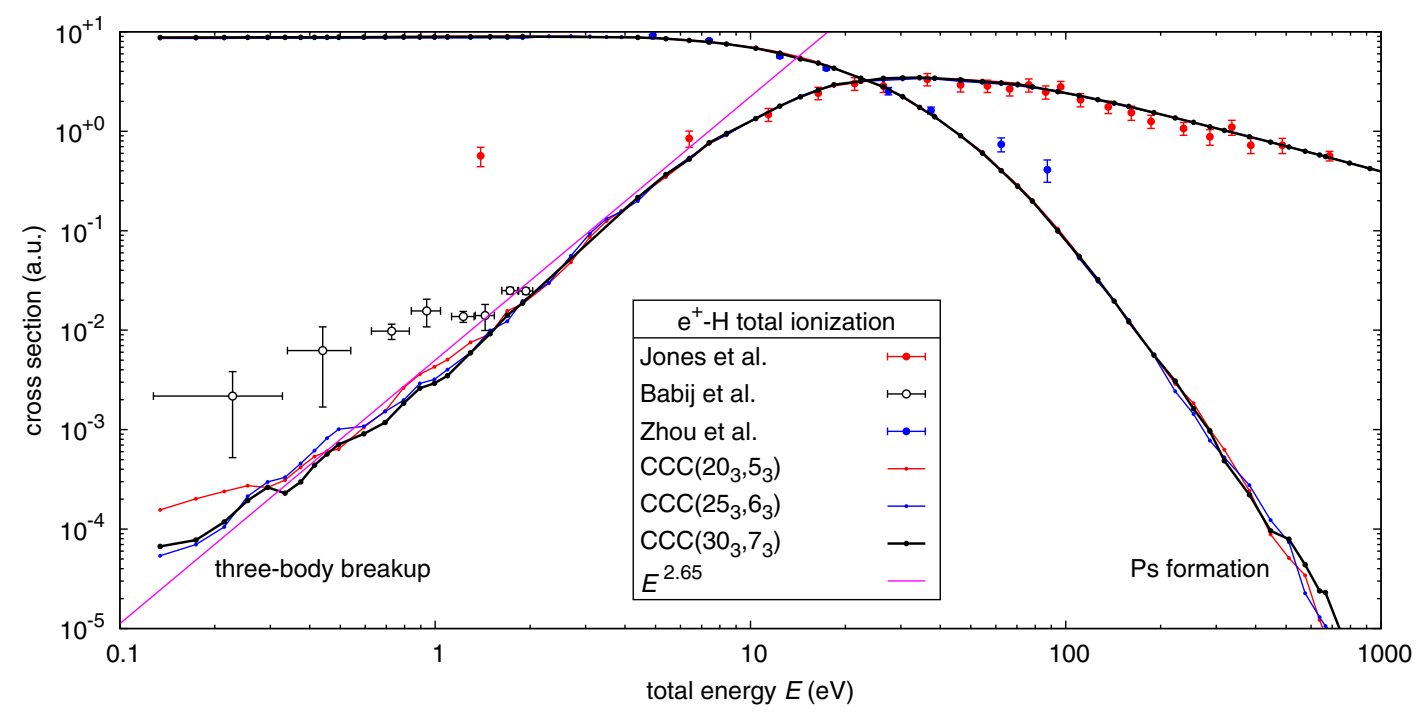

FIG. 2. Total ionization cross sections (Ps formation and three-body breakup) of positron scattering on atomic hydrogen. The present CCC calculations are labeled by $\operatorname{CCC}\left(N_{0 l_{\max }}^{\mathrm{H}}, N_{0 l_{\max }^{\mathrm{Ps}}}\right)$ with $N_{l}=N_{0}-l$ for $l \leq l_{\max }$. The Klar [2] threshold law $\left(\sigma_{I} \propto E^{2.65}\right)$ has been normalized by the best visual fit to the CCC calculations. The experimental data are due to Jones et al. [27] (breakup), Zhou et al. [28] (Ps formation), and Babij et al. [4] (breakup for Argon, normalised at $2 \mathrm{eV}$ ).

contribution to the breakup cross section coming entirely from the positive-energy atomic states. We rely on the completeness of the Laguerre basis on the $\mathrm{H}$ center, complemented by the increasing number of Ps states, to ensure convergence to the correct result.

In Fig. 2 we present three systematically larger CCC calculations performed over the entire energy range from $0.1 \mathrm{eV}$ to $1000 \mathrm{eV}$. They are labeled as $\operatorname{CCC}\left(N_{0 l_{\max }}^{\mathrm{H}}, N_{0 l_{\max }}^{\mathrm{Ps}}\right)$, where the Ps states all have negative energies. We see good agreement between the three calculations except where the cross sections become particularly small. In the case of breakup it is clear that the $E^{2.65}$ suppression of the cross sections as predicted by Klar [2] is being followed by the CCC calculations. It appears that the simple power threshold law is valid for the larger energy range of $\sim 5 \mathrm{eV}$. This coincides with the Ps-formation cross section, a competing ionization process, being constant over this energy range (note the Ps-formation threshold is $E=-6.8 \mathrm{eV}$ ). Apart from the data of Babij et al. [4] agreement with the other measurements is mostly very good over a broad energy range.

In conclusion, using the atomic hydrogen target we have demonstrated that the CCC calculations lend support to the threshold laws of $E^{1.127}$ for electron-impact ionization cross sections to within $\sim 2 \mathrm{eV}$ of threshold where the corresponding spin asymmetries are constant, and $E^{2.65}$ for the positron impact to within $\sim 5 \mathrm{eV}$ where the Ps-formation cross section is constant. Consequently, the CCC calculations do not support the recent measurements or the suggestion of Babij et al. [4] that the electron-impact and positron-impact near-threshold ionization cross section behavior is the same.
We acknowledge the support of the Australian Research Council, and the resources and services from the National Computational Infrastructure and the Pawsey Supercomputer Centre, which are supported by the Australian and Western Australian Governments.

[1] G. H. Wannier, Phys. Rev. 90, 817 (1953).

[2] H. Klar, J. Phys. B 14, 4165 (1981).

[3] W. Ihra, J. H. Macek, F. Mota-Furtado, and P. F. O’Mahony, Phys. Rev. Lett. 78, 4027 (1997).

[4] T. J. Babij, J. R. Machacek, D. J. Murtagh, S. J. Buckman, and J. P. Sullivan, Phys. Rev. Lett. 120, 113401 (2018).

[5] P. Ashley, J. Moxom, and G. Laricchia, Phys. Rev. Lett. 77, 1250 (1996).

[6] A. Rau, Phys. Rep. 110, 369 (1984).

[7] J.-M. Rost, Phys. Rep. 297, 271 (1998).

[8] A. Temkin, Phys. Rev. Lett. 49, 365 (1982).

[9] A. Temkin, J. Phys. B 15, L301 (1982).

[10] R. Peterkop, J. Phys. B 16, L587 (1983).

[11] P. L. Bartlett, A. T. Stelbovics, and I. Bray, Phys. Rev. A 68 , 030701(R) (2003).

[12] G. D. Fletcher, M. J. Alguard, T. J. Gay, V. W. Hughes, P. F. Wainwright, M. S. Lubell, and W. Raith, Phys. Rev. A 31, 2854 (1985).

[13] D. M. Crowe, X. Q. Guo, M. S. Lubell, J. Slevin, and M. Eminyan, J. Phys. B 23, L325 (1990).

[14] I. Bray and A. T. Stelbovics, Phys. Rev. A 46, 6995 (1992).

[15] A. S. Kadyrov and I. Bray, Phys. Rev. A 66, 012710 (2002).

[16] I. Bray, I. B. Abdurakhmanov, J. J. Bailey, A. W. Bray, D. V. Fursa, A. S. Kadyrov, C. M. Rawlins, J. S. Savage, A. T. Stelbovics, and M. C. Zammit, J. Phys. B 50, 202001 (2017).

[17] I. Bray, Phys. Rev. Lett. 89, 273201 (2002). 
[18] I. Bray and A. T. Stelbovics, Phys. Rev. Lett. 70, 746 (1993).

[19] A. S. Kadyrov, I. Bray, A. M. Mukhamedzhanov, and A. T. Stelbovics, Ann. Phys. (Amsterdam) 324, 1516 (2009).

[20] I. Bray, D. V. Fursa, A. S. Kadyrov, A. T. Stelbovics, A. S. Kheifets, and A. M. Mukhamedzhanov, Phys. Rep. 520, 135 (2012).

[21] M. B. Shah, D. S. Elliot, and H. B. Gilbody, J. Phys. B 20, 3501 (1987).

[22] I. Bray, J. Phys. B 28, L247 (1995).

[23] I. Bray, Can. J. Phys. 74, 875 (1996).
[24] A. S. Kadyrov and I. Bray, J. Phys. B 49, 222002 (2016).

[25] A. W. Bray, I. B. Abdurakhmanov, A. S. Kadyrov, D. V. Fursa, and I. Bray, Comput. Phys. Commun. 203, 147 (2016).

[26] A. S. Kadyrov, I. Bray, and A. T. Stelbovics, Phys. Rev. Lett. 98, 263202 (2007).

[27] G. O. Jones, M. Charlton, J. Slevin, G. Laricchia, A. Kövér, M. R. Poulsen, and S. N. Chormaic, J. Phys. B 26, L483 (1993).

[28] S. Zhou, W. E. Kauppila, C. K. Kwan, and T. S. Stein, Phys. Rev. Lett. 72, 1443 (1994). 